\title{
Research of the Marine Delimitation Information System based on the ArcGIS Engine
}

\author{
Zhang Jie ${ }^{1, a}$, Zhang Jianhui ${ }^{* 1, b}$,Jiang Bing ${ }^{1, c}$ \\ ${ }^{1}$ National Marine Data and Information Service, LiuWei Road 93", HeDong district, TianJin, China \\ a191490839@qq.com, bjh_011@163.com, ${ }^{\mathrm{c}} 250560161 @ q q . c o m$
}

\begin{abstract}
Keywords: Marine Delimitation, Emergency analysis, Maritime Right.
Abstract. The maritime right of our country faces severe situation, there is complicated work of marine delimitation between peripheral marine countries and our country, the research on marine delimitation technology using of information technology is an important task. This paper has analyzed the urgency and feasibility of GIS technology to build maritime delimitation information system, proposed the feature, goal and principle of the system, designed system overall architecture and applied ArcGIS Engine component, interface and three dimensional ellipsoid measurement technology to develop to achieve the related functions of system.
\end{abstract}

\section{Introduction}

The 21 st century is marine century. Covering $71 \%$ surface of the earth, the ocean is not only the fundamental component of the global life supporting system but also the resource treasury, as well as the important regulator of environment ${ }^{[1]}$.

With the development of economic society and the depletion of land resource, the coastal counties start to turn to the ocean for resource exploitation. Especially as the "United Nations Convention on the Law of the Sea" came into force on November 16, 1994 and the extensions of the relevant countries' governing sea area, the marine rights and interests and safety of our country have met the increasing challenges from peripheral coastal states and other ocean powers ${ }^{[2]}$. It affects states relation when territorial sovereignty are infringed, islands and reefs are invaded by other countries and the conflict of maritime comes to surface and regular. Apparently, the momentum of development is more and more serious, it will be a long term struggle to safeguard maritime rights and interests ${ }^{[3]}$.

As an emerging advanced technology, Geographic Information System has been quickly applied in various industries where remarkable achievements have been made ${ }^{[4]}$. By using of geographic information technology, computer network and database technology, the building of maritime delimitation information system based on GIS will help achieve the digital management and sharing of maritime delimitation information, enhance the analysis and disposal capacity to deal with emergency rights and interests event, promote the safeguard work of maritime rights and interests.

\section{The goal, content and principle of system}

Using GIS and space database technology, the goal of establishing the maritime delimitation information system is to build one comprehensive information platform with the rich materials, accurate analysis and calculating, and full and practical functions, which can provide information service and decision support for the safeguard of maritime rights and interests and maritime delimitation.

The content of the system construction includes the designing and building of marine basic geographic information database, marine rights and interests professional geographic information database, maritime rights and interests of business information database, maritime rights and interests of emergency resource information database and maritime rights and interests of background information database in order to realize the integrated storage and management of geospatial data and business attribute data. Based on ArcGIS Engine components technology, the system should achieve spatial visualization analysis, representation and output of emergency event of maritime rights and 
interests. Based on three dimensional ellipsoid measurement technology, the platform should realize the maritime delimitation function such as emergency case analysis of rights and interests, analyzing and calculating and automatically derivation of equidistant/medium line, equiratio line, latitude bisector, longitude polygon bisector and provide assistant decision-making for the maritime delimitation work.

According to the goal and content of the system establishment, the following principles should be strictly kept to:

(1) Practicality principle: the system design satisfies business requirement, the function is pragmatic, interface is friendly and the operation is simple.

(2)Advancement principle: in the aspect of system function and algorithm design and program development, the system construction should take advantage of state of art software development technology, the GIS platform and the third-part plug-in should choose the leading technology and mature products.

(3)Reliability and the security principle: all the calculation and analysis results of system should be precious, reliable and satisfy the accuracy requirements. The system operation should be highly continuous, stable and secure.

(4)Economic Principle: under the premise of ensuring leading technology, we should take into account the economic system construction, and make the most of the current technology and resource to save the construction costs.

(5)Open and expansibility principle: taking the possibility of changes in business requirement and service mode into account and applying the open design. Not only to conclude integrity and openness, but also the scalability of system, which makes the system facile to maintain and upgrade.

\section{The design of system architecture}

The entire system architecture uses C/S mode. The overall system architecture is divided into five layers including the data layer, the service layer, the function layer, the application layer and the user layer from the bottom up. The system architecture is shown in figure 1 .

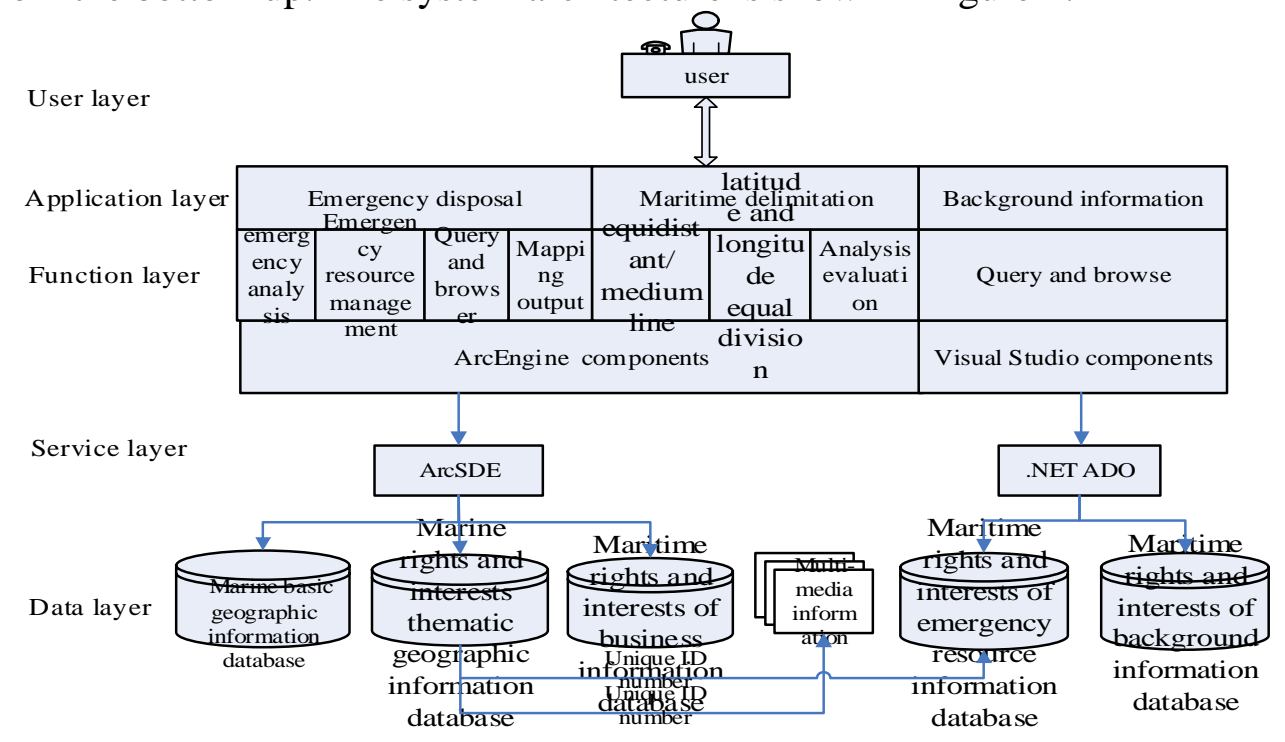

Fig.1 The system architecture

The data layer is the important building block and foundation of the entire system and all the inquiry browsing and calculation analysis of system are conducted on the data layer. It consists of the maritime basic geographic information database, maritime rights and interests thematic geographic information database, business information database of maritime rights and interests, maritime emergency resource information database and maritime rights and interests of background information database. 
The maritime basic geographic information database includes the global marine fundamental spatial data at the scale of 1:10000000, 1:5000000 and 1:1000000 etc. Remote sensing data uses the global remote sensing image data at a scale of 1:10000000. The marine rights and interests professional geographic information database includes the geographic information of the basepoint, baseline and boundary line of territorial sea, the line of exclusive economic zone, maritime boundaries, fishing zone, joint development area and bidding area of our and surrounding countries. The business information database of maritime rights and interests includes the related laws and regulations, treaty and chronicle of events etc. The maritime emergency resource information database includes the emergency plan and strategy and handling specialist, institution, team and equipment. The background information database include the relative full information of maritime rights and interests work such as literature materials, books and journals.

The backstage database of system uses SQL Server 2005. The system uses the ArcSDE middleware to storage and manage all the spatial data and business data of maritime rights and interests. The old massive attribute data can be stored in SQL Server 2005 database directly. The multimedia information can be stored in a fixed position of server in folder.

The service layer mainly provides component, interface or middleware service to system function layer. The service layer is the foundation of system function layer. Basing on this layer, all application functions of system including inquiry and analysis of GIS function and full-text information browsing function can be realized. The service layer accesses the data layer in two ways. For geospatial data and business data, it employs the ArcSED middleware to unified management, and the relative multimedia data and external attribute data are realized association operation by unique ID number. For external attribute data, it uses NetADO to realize the inquiry and management.

The function layer achieves the relative functions according to the user requirements based on the interface, component and service provided by service layer, and mainly includes functions such as emergency analysis, emergency resource management, query statistics, equidistant/medium line delimitation, latitude and longitude equal division polygon delimitation and the query browser of background information.

The system application layer is mainly designed through the research and requirement analysis of end user, summarizing the use's application direction which can be expressed by the computer system in abstract. The applications of this system includes emergency disposal, maritime delimitation and background information three aspects.

The user layer is the end user that proposes the system application requirements.

\section{The function of system}

The maritime delimitation information system mainly includes emergency disposal, maritime delimitation and background information management three functions.

\section{Emergency handling}

(1) Emergency analysis: emergency analysis is the core function of this system. Through the map spatial positioning of emergency event of maritime rights and interests, it firstly automatically calculates the distance and position between current position and the specified maritime boundary and then matches and provides the relative plan in the system emergency resource database if there is one, according to the event position, nature of incident and event level. Simultaneously, according to need, the emergency analysis will retrieve the important channel, offshore installations and restricted zones of incident location in the map, carry on correlation analysis, form one thematic map and provide the related user with the visual analysis results. The schematic diagram of distance and azimuth of a maritime incident position and surrounding countries is shown in figure 2. 


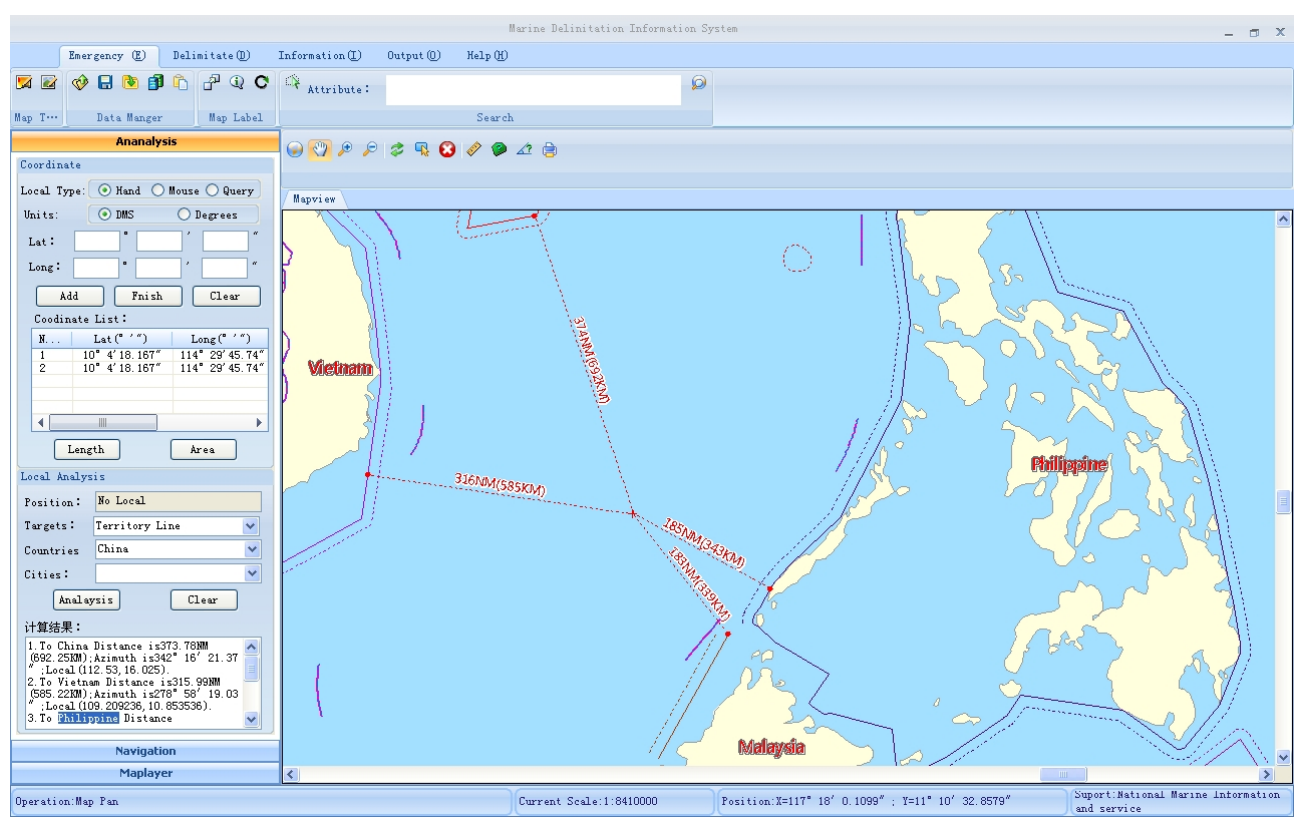

Fig.2 Emergency analysis

(2) Emergency resource management: it is mainly focused on realizing the management of necessary emergency resource information handling of all kinds of maritime rights and interests of emergency incident. The emergency resource information includes emergency plan information, emergency specialist information, emergency handling team information and emergency equipment information.

According to the geographical location, nature and level of emergency incident of maritime rights and interests, the system implements the effective query, browsing and management of each type emergency resource information, and provides the related user with scientific decision of handling emergency incident.

(3) Information query: system information query includes two ways, the condition query and the map spatial query.

The condition query implements spatial information query retrieval by entering keyword that satisfied condition, and put the results into the query list. The spatial query is achieved by choosing the range of map with the mouse, and system automatically retrieve the elements in this range, and display the results in the query list. Meanwhile, it can realize the position of factor space by double-clicking record in the query result list, and browse the basic information, picture information and video information.

(4) Case statistics: the function of case statistic mainly make statistical analysis of rights and interests cases according to region in which case happened, case category and case level three methods in a period of time and the statistical results are showed in various angles and situations of report, statistical graph and thematic maps. This can provide the decision basis for the coming maritime safeguard rights in the future.

(5) Mapping output: for the incident of rights and interests, the system emergency analysis function analyzes and calculates the distance and position from the location of incident to the target information of different countries. The system marking function plots descriptive information such as characters and symbol on the map. Adding the elements of map layout such as title of map, mapping unit, map grid, compass and map scale can achieve the cartography and map output on the publication level.

\section{Maritime delimitation}

Using the component interfaces and earth ellipsoid calculating technology of ArcGIS Engine, the system implements the method of high precision of maritime delimitation in three dimensional surface of the earth, and includes methods such as equidistant/medium line, equal proportion, latitude bisector, longitude polygon bisector. 
The first step of the maritime delimitation process is preparation of delimitation data in general. The delimitation data must be the geographic data obtained accreditation by both sides and includes data coordinate system, datum plane, scale, coordinate precision and properties of line and surface. Second, the data including delimitation sea area, baseline used to delimitation and associated evaluation data can be imported into the system and then the system can be employed to provide the methods to carry out the scheme line calculation.

The system also can analyze and evaluate the divided waters according to the generated scheme line, calculate the range of water of two sides and the total amount and percentage of oil and gas in quantum, and provide technology support for making delimitation scheme. The delimitation scheme line of equal proportions value is 3 between country A and country B is shown in figure 3 .

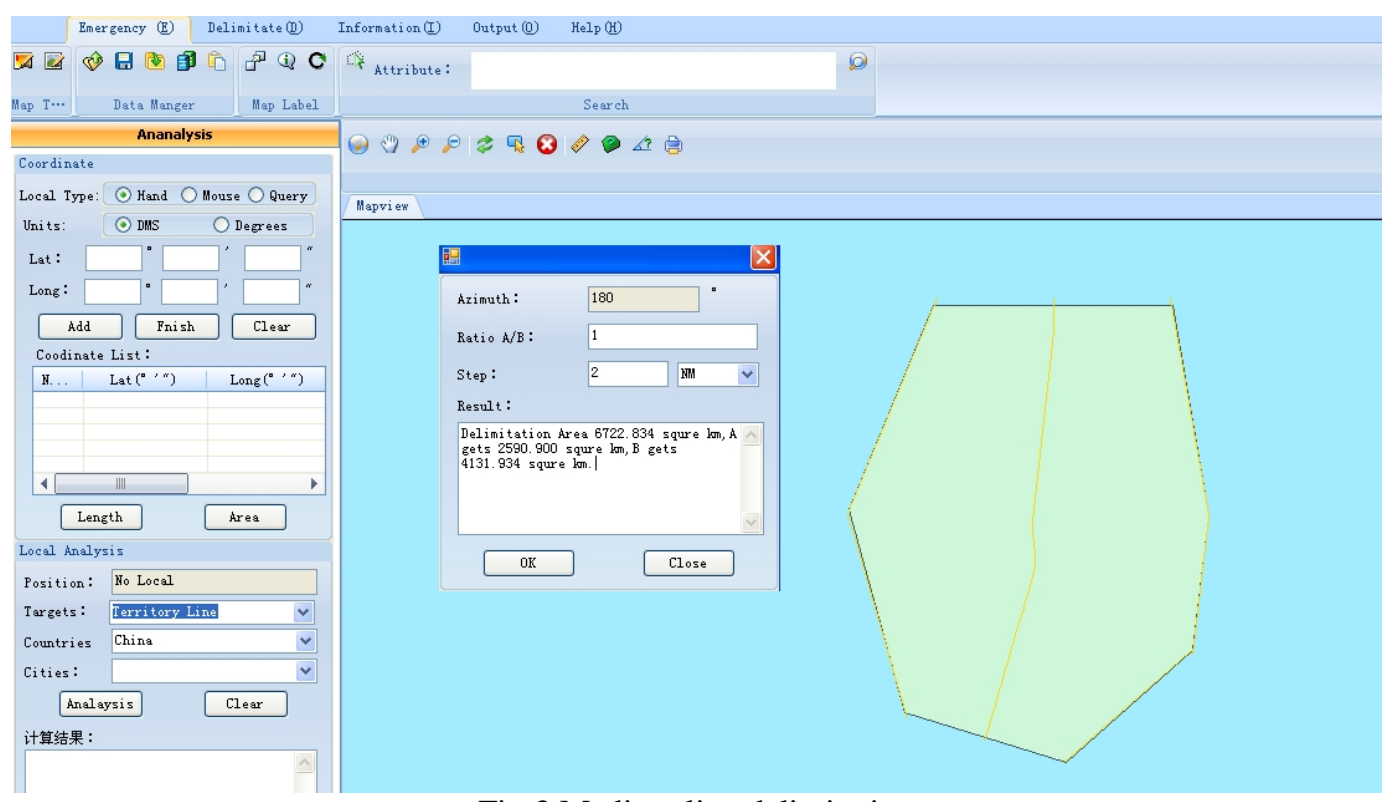

Fig.3 Medium line delimitation

\section{Background information management}

Background information module mainly achieve the managements of variety of literatures and factual information related to the maintenance of maritime rights and interests, including laws and regulations, delimitation case of and chronicle. The function of this module can realize target information querying and full-text browsing.

\section{Conclusion}

This paper has studied the implement of the integrated storage and management of maritime delimitation information spatial data and business data by using spatial database technology. Based on three dimensional ellipsoid measurement technology, realize thee emergency analysis, spatial measure analysis and maritime delimitation function, and preferably solve the excessive error problem of calculating long distance, large area and angle in general GIS platform, and meet for the maintenance work of maritime rights and interests.

\section{References}

[1] Ye Xiangdong, Maurice chan. Construction of "digital ocean" implementation plan as a whole the land and sea [J]. Journal of Pacific Ocean, 2007, 4:77-86.

[2] Li Sihai, Jin Jiye. Maritime rights and interests maintenance information system [J]. The design and implementation of Marine surveying and mapping, 2006, 26 (3) : 55-58.

[3] Wu Jiang, Zhao Shengru. Maritime rights and interests maintenance enforcement countermeasure analysis [J]. Journal of ocean development and management, 2004, 6:38 and 43. 
[4] James zhang, Chunlin Xia, Cui Yanjun etc. Based on the technology of image engine JieChuJing GIS system design and implementation of [J]. Bulletin of surveying and mapping, 2008, 2:59-61. 